\section{Chloride channels revisited}

\section{Chris Higgins}

Cystic fibrosis (CF) is one of the most common serious inherited diseases among caucasians. The main clinical symptoms are associated with epithelial tissues, particularly those of the lung, pancreas and intestine. One of the challenges of $\mathrm{CF}$ research has been to identify the primary defect responsible for these symptoms. The characterization of the gene at fault in CF, and the demonstration that its product, the $\mathrm{CF}$ transmembrane conductance regulator (CFTR), is a chloride channel, have ensured that we are well on our way to an answer ${ }^{1-3}$. But these elegant molecular studies are only part of the CF story. The function of CFTR now has to be understood in terms of epithelial physiology. What is the role of the CFTR chloride channel in relation to other epithelial ion channels? How does a defect in CFTR result in the many other biochemical differences between CF and non-CF cells and tissues? And, crucially, how does a defect in CFTR result in the pathophysiology of the disease? A first step towards these long-term goals is suggested in a report by Egan et al. on page 581 of this issue ${ }^{4}$.

It is now almost ten years since altered epithelial ion conductances were first associated with cystic fibrosis ${ }^{5}$. Epithelial cells contain many apparently independent ion channels and, with a view to pharmacological intervention, considerable effort has been invested in identifying and characterizing the specific ion channel(s) associated with CF. A few years ago a chloride channel, the socalled 'outwardly rectifying chloride channel' (ORCC) emerged as a strong candidate for the primary defect in $\mathrm{CF}^{6-8}$. Several independent research groups reported that this channel could not be activated by either protein kinase A or protein kinase $\mathrm{C}$ in membranes from CF cells (in contrast to membranes from non-CF cells where it could be activated). As the ORCC could be activated in $\mathrm{CF}$ membranes by 'nonphysiological' procedures, for example by large depolarizing voltage pulses, it was concluded that the channel itself was present in CF cells but that its regulation was defective.

This was the situation in 1989 when the CF gene was identified. As CFTR had all the characteristics of a membrane protein, it raised the possibility that CFTR might itself be the ORCC. Subsequently CFTR was indeed shown to be a chloride channel ${ }^{2,3}$. But $\mathrm{CF}$ research has never been that simple: the properties of the CFTR channel were shown to be entirely different from those of the
ORCC. Furthermore, it became clear that expression of CFTR does not correlate with the presence of ORCC, and vice versa ${ }^{9}$. As requested in Nature at that time ${ }^{10}$, "Will the real $\mathrm{Cl}^{-}$channel please stand up?". Researchers had to conclude either that CFTR can regulate the ORCC (an unwanted complication in the early flush of excitement following the initial characterization of the $\mathrm{CF}$ gene product), or that earlier conclusions regarding the ORCC were 'unfortunate'. The role of the ORCC in CF was quietly forgotten.

The report by Egan and colleagues ${ }^{4}$ suggests that the ORCC should be resurrected. Their data support the conclusion that CFTR and the ORCC are distinct chloride channels, but the authors also present important evidence that CFTR can regulate the ORCC. Thus in cells lacking CFTR, the ORCC cannot be activated by protein kinase $A$; when CFTR is expressed in these cells, the ability of protein kinase A to activate the ORCC is restored. So here we have a potential reconciliation of 'pre-CF gene' data on the ORCC with the 'post$\mathrm{CF}$ gene' studies on CFTR. But, more significantly, these studies provide direct evidence that CFTR can influence the activity of another ion channel. There are many, apparently independent, ion channels in epithelial cells, and both sodium and chloride conductances seem to be altered in CF. If CFTR can regulate the activity of other epithelial ion channels it is, perhaps, easier to understand how loss of CFTR, a smallconductance chloride channel, can lead to the considerable changes in transepithelial ion conductances that have been measured in membranes from $\mathrm{CF}$ cells.

How could the CFTR regulate the ORCC? Perhaps least satisfying would be by an indirect effect. It is known that the principal mutant form of CFTR does not reach the apical membrane (where CFTR is normally located) but is incorrectly glycosylated and remains in the Golgi: this may, fortuitously, influence the traffic of other proteins (perhaps the ORCC) to the apical membrane ${ }^{11,12}$ The more enticing possibility is that the effect of CFTR expression on the ORCC reflects a physiological function of CFTR. This would be of considerable importance for our understanding of CF.

Several mechanisms by which CFTR might regulate the $\mathrm{ORCC}$ can be envisaged. It could interact directly with the ORCC protein in the apical membrane of epithelial cells (note that we do not yet know the protein responsible for the
ORCC). But as the CFTR channel is activated by protein kinase $\mathrm{A}$ and protein kinase A is necessary for CFTR to influence the ORCC, it seems more likely that the effect of CFTR on the ORCC is a consequence of CFTR function. CFTR is a chloride channel and the movement of chloride through CFTR could, potentially, regulate the ORCC (although it is not easy to envisage how). Alternatively, the regulation of the ORCC by CFTR might reflect a function for CFTR in addition to its channel activity; intriguingly, the closely related multidrug-resistance P-glycoprotein is bifunctional, being associated with both chloride channel and active transport activities $^{13,14}$. The experimental tools are now available for these options to be tested critically.

What are the consequences of these findings for CF therapy? The observation that, in most cases of CF, the mutant CFTR protein is incorrectly localized (it does not get to the apical membrane of epithelial cells) makes it difficult to contemplate restoring the defective protein by conventional pharmacological intervention; instead, effort has focused on replacing the mutant CFTR by gene or protein therapy. If, however, the pathophysiological changes in $\mathrm{CF}$ are, at least to some extent, a consequence of the failure to regulate other epithelial ion channels rather than of the absence of the small-conductance chloride channel per se, pharmacological intervention to restore regulation to these other channels becomes an option. Evidently several important questions still remain to be answered in the wake of the quantum leap in our understanding generated by the detailed analysis of the $\mathrm{CF}$ gene and its product over the past three years. What is, perhaps, most exciting is that studies of cystic fibrosis not only hold out promise for treatment of the disease, but also continue to provide deep and general insights into cell biology, biochemistry and physiology.

Chris Higgins is at the ICRF Laboratories, Institute of Molecular Medicine, University of Oxford, John Radcliffe Hospital, Oxford OX3 9DU, UK.

\footnotetext{
1. Riordan, J. R. et al. Science 245, 1066-1073 (1989).

2. Anderson, M. P. et al. Science 251, 679-682 (1991)

3. Bear, C. E. et al. Cell 68, 809-818 (1992).

4. Egan, M. et al. Nature 358, 581-584 (1992)

5. Quinton, P. M. Nature 301, 421-422 (1983)

6. Schoumacher, R. A. et al. Nature 330, 752-754

(1987).

7. Li, M. et al. Nature 331, 358-360 (1988)

8. Hwang, T.-C. et al. Science 244, 1351-1353 (1989).

9. Ward, C. L. et al. Proc. natn. Acad. Sci. U.S.A. 88 5277-5281 (1991)

10. Frizzell, R. A. \& Cliff, W. H. Nature $350,277-278$ (1991)

11. Cheng, S. H. et al. Cell 66, 1027-1036 (1991)

12. Bradbury, N. A. et al. Science 256, 530-532 (1992)

13. Valverde, M. A. et al. Nature 355, 830-833 (1992).

14. Gill, D. R. et al. Cell (in the press)
}

NATURE · VOL 358 • 13AUGUST 1992 